\title{
4 Der Einfluss des Klimawandels auf das Auftreten von Herz-Kreislauf-Erkrankungen. Handlungsansätze und die besondere Herausforderung durch Arzneimittel- wechselwirkungen
}

\author{
Bernhard Kuch
}

C. Günster || Klauber | B.P. Robra | C. Schmuker | A. Schneider (Hrsg.) Versorgungs-Report Klima und Gesundheit. DOI 10.32745/9783954666270-4, ( MWV Medizinisch Wissenschaftliche Verlagsgesellschaft Berlin 2021

Für die Bevölkerung am augenscheinlichsten und unmittelbar auch am Körper spürbar ist die mit dem Klimawandel einhergehende Zunahme der Hitzeperioden. Diese haben unmittelbare negative Auswirkungen auf die Herzinfarktmorbidität und -mortalität, aber auch auf Schlaganfallhäufigkeit und andere Akutmanifestationen von Herz-Kreislauf-Erkrankungen. Besonders gefährdet sind Patienten in höherem Lebensalter und mit vorbestehenden Komorbiditäten. Zum Schutz der Bevölkerung vor hitzebedingten Gesundheitsschäden müssen verschiedene präventive Maßnahmen koordiniert umgesetzt werden. Dazu zählt (ganz allgemein) eine verbesserte Edukation über die Auswirkungen von Hitzeperioden auf die Gesundheit in der Ausbildung der Gesundheitsfachberufe. Maßgeblich für die Behandlung von Risikopatienten ist zudem die Vermittlung und Kenntnis von hitzebedingten Arzneimittelwechselwirkungen. Als eine zielgerichtete Maßnahme hierfür wird die Entwicklung und Implementierung eines Smartphonebasierten Ampelsystems vorgeschlagen, welches Patienten und die behandelnden Ärzte über gesundheitsgefährdende Wetterlagen und die Einnahme von kritischen Medikamenten informiert.
In people the most obvious and directly noticeable impact on their body associated with climate change are the increase in heat periods. These also have immediate negative effects on heart attack morbidity and mortality, but also on stroke frequency and other acute manifestations of cardiovascular diseases. Patients with higher age and with pre-existing co-morbidities, especially patients with diabetes and heart failure, are particularly at risk. Preventive measures to protect against heat-related health damage can be undertaken through coordinated activities to prevent population impacts, improved education on the effects of heat periods on health in training and mediation in health professional. It warrants in addition targeted measures, such as the implementation of a smartphonebased traffic light system with, in the event of heat periods, timely warning all at-risk patients or their relatives or doctors and transmitting generally appropriate heat protection measures and flashing of checklists that query the treatment with critical medications. 


\section{Fallbeispiel}

Der Notarzt wird an einem Juliabend zu einer 77-jährigen Frau gerufen, da diese für ca. 3 Minuten bewusstlos zusammengesackt war. Die Tage zuvor habe sie viel im Garten gesessen und bei der gegenwärtigen Hitzeperiode (über drei Tage Spitzentemperaturen über $30 \mathrm{Grad}$ Celsius) viel geschwitzt, sie habe wohl auch zu wenig getrunken. Bei Eintreffen des Notarztes und auch in der Klinik war die Patientin bewusstseinsklar und in stabilem Herz- und Kreislaufzustand. Das Labor zeigte bis auf eine mäßiggradige Kreatininerhöhung $(1,8 \mathrm{mg} / \mathrm{dl})$ keine relevanten Auffälligkeiten. Der Röntgen-Thorax-Befund zeigte eine Linksherzvergrößerung ohne aktuelle Stauungszeichen, echokardiografisch zeigte sich eine linksventrikuläre Hypertrophie und eine mittelgradig eingeschränkte Pumpfunktion (EF ca. 40\%). Die weitere Vorgeschichte erbrachte einen langiährigen arteriellen Hypertonus, die Medikation bestand aus: Sotalex (Sotalol, einem Betablocker und Klasse-III-Antiarrhythmikum) $2 \times 160 \mathrm{mg}$, Isoket ret. (ein Nitrat) $2 \times 20 \mathrm{mg}$, ASS $100 \mathrm{mg}$ und HCT (Hydrochlorothiazid, ein Diuretikum) $25 \mathrm{mg}$. Die Patientin wurde zunächst auf der Nachtstation überwacht. Um 2.00 Uhr morgens trat eine erneute Synkope auf und die Patientin musste aufgrund eines Kreislaufstillstandes reanimiert werden. Im EKG zeigte sich eine Torsade de pointes-Tachykardie (Spitzenumkehrtachykardie) mit Übergang in Kammerflimmern. Nach einmaliger Defibrillation konnte ein Sinusrhythmus und wieder ein stabiler Kreislaufzustand erreicht werden, zur weiteren Überwachung wurde die Patientin auf die Intensivstation verbracht, nach Infusion einer Magnesiumsulfatlösung traten keine weiteren Rhythmusstörungen mehr auf. Das Aufnahme-EKG, welches initial als reines „Hypertrophie-EKG“ gewertet wurde, zeigte eine signifikante QT-Zeitverlängerung. Die abschließende Diagnose lautete daher: Rezidivierende Synkopen mit letztlich Reanimation bei Kammerflimmern bei Torsade de Pointes-Tachykardien bei pathologischer QT-Zeitverlängerung unter Therapie mit Sotalol bei relativer Überdosierung im Rahmen einer exsikkosebedingten Nierenfunktionsstörung. Letztere war ausgelöst durch vermehrtes Schwitzen und unzureichende Flüssigkeitszufuhr im Rahmen der Hitzeperiode. Sotalol wurde abgesetzt und durch Bisoprolol (einen reinen Betablocker) ersetzt und die Patientin instruiert, bei ent- sprechenden Umgebungsbedingungen auf ausreichende Flüssigkeitszufuhr zu achten und Schutz in abgekühlten Räumen zu suchen. Im Arztbrief an die weiterbehandelnden Ärzte wurde auf die Gefährlichkeit von Klasse-III-Antiarrhythmika, die, wie Sotalol, vornehmlich über die Niere ausgeschieden werden, hingewiesen. Zudem wurde gebeten, bei Anwendung von Diuretika in Hitzeperioden die Patientin engmaschiger klinisch und laborchemisch zu kontrollieren.

\subsection{Einleitung}

Für die Bevölkerung am augenscheinlichsten und unmittelbar auch am Körper spürbar ist die mit dem Klimawandel einhergehende Zunahme der Hitzeperioden. So berichtet der im November 2019 in Berlin vorgestellte Jahresbericht der internationalen Forschungsinitiative „Lancet Countdown“ über bis zu fünf zusätzliche Hitzewellen in Norddeutschland und bis zu 30 zusätzliche Hitzewellen in Süddeutschland jeden Sommer (Jacob et al. 2014). Die internationale Forschungsinitiative „Lancet Countdown“ entstand über die Zeitschrift „The Lancet“ und war als globales Monitoring-System gedacht. Seit der Veröffentlichung des ersten Berichts im Jahr 2015 wird jährlich über den Klimawandel und seine Auswirkungen auf die Gesundheit berichtet (Watts et al. 2015). Zudem wird ein Anstieg der Durchschnittstemperatur um $3,7^{\circ} \mathrm{C}$ für die kommenden Jahrzehnte prognostiziert (Deutscher Wetterdienst 2020).

Kapitel 1 und 2 in diesem Buch befassen sich umfassend mit den Folgen des Klimawandels auf die Umwelt- und Lebensbedingungen. Im Kapitel 3 wurde der Einfluss der mit dem Klimawandel einhergehenden Temperaturveränderungen auf die Mortalität beschrieben. Wir erläutern im Folgenden zunächst die bekannten wissenschaftlichen Erkenntnisse über den Einfluss der Temperaturänderungen auf die HerzKreislauf-Morbidität anhand von Studien aus der Region Augsburg. Vertiefend wird dann auf die Herausforderungen durch hitzebedingte Arzneimittelwechselwirkungen eingegangen. 


\subsection{Einfluss von Hitzewellen auf die Herzinfarkthäufigkeit und das Auftreten anderer Kreislauferkran- kungen - Evidenz aus Augsburg}

Das Augsburger Herzinfarktregister sammelt seit 1985 als Beteiligung am WHO MONICA-Projekt sämtliche Herzinfarktfälle bzw. im prähospitalen Setting Verdachtsfälle auf Herzinfarkt. Es werden damit alle potenziellen Herzinfarktfälle der 25- bis 84-jährigen (bis 2008 74-jährigen) Einwohner der Stadt Augsburg und der angrenzenden Landkreise Augsburg und AichachFriedberg erfasst. Es wird dabei die Herzinfarktmorbidität mit allen tödlichen und nichttödlichen Herzinfarkten, inklusive der plötzlichen Herztodesfälle je 100.00o Einwohner ermittelt, zusätzlich die Mortalität und 28-Tage-Letalität. Es wird hierbei besonders unterschieden zwischen der Letalität in der Phase vor Erreichen einer Klinik (prähospitale Phase) und der Letalität im weiteren hospitalen Verlauf. Diese Form der Datenerhebung, insbesondere auch unter Einbeziehung aller prähospitalen Todesfälle, mit der Möglichkeit, dies auch mit Klimadaten zu verbinden, ist in Deutschland einmalig.

So konnten Chen et al. anhand von Daten des Augsburger Herzinfarktregisters zeigen, dass starke Temperaturausschläge das Risiko für einen Herzinfarkt erhöhen können (2019a): Einbezogen in die Analyse wurden über 27.0oo Herzinfarktfälle zwischen 1987 und 2014. Das Durchschnittsalter der Betroffenen betrug rund 63 Jahre, $73 \%$ davon waren Männer und rund 13.000 Fälle endeten tödlich. Hierzu wurden im Rahmen des Augsburger Herzinfarktregisters alle in der Region aufgetretenen tödlichen und nicht-tödlichen Herzinfarkte erfasst. Die einzelnen Infarkte wurden gemeinsam mit den meteorologischen Daten des jeweiligen Tages und der vorangegangenen Tage verknüpft, und mögliche andere Faktoren wie beispielsweise Wochentage, klassische Risikofaktoren oder sozioökonomischer Status herausgerechnet. Es wurden die Ergebnisse aus dem Zeitraum 1987 bis 2000 mit denen zwischen 2001 und 2014 verglichen und gezeigt, dass das Herzinfarktrisiko mit zunehmender täglicher Durchschnittstemperatur stärker ansteigt als im vorangegangenen Untersuchungszeitraum . Dieses Ergebnis war Ausgangspunkt für eine weitere Analyse der Autoren. Chen et al. prognostizierten unter Verwendung von täglichen Herzinfarkt-Zeitreihen und Temperaturprojektionen basierend auf zwei Klimaszenarien jeweils die Zahl der temperaturbedingten Herzinfarkte bei unterschiedlichen Erwärmungsausprägungen (Chen et al. 2019b). Es wurde gezeigt, dass in einem emissionsarmen Szenario, das die Erwärmung im 21. Jahrhundert auf unter $2^{\circ} \mathrm{C}$ begrenzt, die Zahl der temperaturbedingten Herzinfarkte bei einer globalen Erwärmung um $1,5^{\circ} \mathrm{C}$ mit - 6 Fällen (95\%-Konfidenzintervall: $[-60 ; 50]$ ) pro Jahrzehnt (Nettoveränderung durch Berücksichtigung von kälte- und wärmebedingten Belastungen) leicht abnehmen wird. In einem Szenario mit hohen Emissionen, welches die Ziele des Übereinkommens von Paris überschreitet, steigt pro Jahrzehnt die Zahl der temperaturbedingten Herzinfarkte bei Erwärmung um $2^{\circ} \mathrm{C}$ um 18 [-64; 117] beziehungsweise bei Erwärmung um $3^{\circ} \mathrm{C}$ um $63[-83$; 257] Fälle an. Zwar sind die Schätzungen zur Nettoveränderung künftiger temperaturbedingter Herzinfarktereignisse nicht signifikant, die Schätzungen bezüglich hitzebedingter Herzinfarkte sind jedoch statistisch signifikant und zeigen einen Anstieg von 54 (95\%-Konfidenzintervall: $[1 ; 124])$ beziehungsweise 109 [4; 313] Fällen pro Jahrzehnt bei einer globalen Erwärmung um $2^{\circ} \mathrm{C} / 3^{\circ} \mathrm{C}$. Die nichtsignifikanten Schätzer der Nettoveränderung sind vorwiegend auf die Unsicherheit bei den Schätzungen der kältebedingten Belastung zurückzuführen. Dies legt nahe, dass bei einer Erwärmung um $1,5^{\circ} \mathrm{C}$ geringere Risiken für hitzebedingte Morbidität und Mortalität prognostiziert werden als bei einer Erwärmung um $2^{\circ} \mathrm{C}$ (Chen et al. 2019b). Das Fazit der Autoren lautet, dass die künftige Belastung durch temperaturbedingte Herzinfarkt-Fälle in Augsburg bei einer 
II Gesundheitliche Auswirkungen des Klimawandels und Herausforderungen

für die medizinische Versorgung in Deutschland

globalen Erwärmung um $2^{\circ} \mathrm{C}$ beziehungsweise $3^{\circ} \mathrm{C}$ höher liegen wird als bei einer Erwärmung um $1,5^{\circ} \mathrm{C}$. Schlussfolgerung aus der Studie war, dass die Erfüllung des Übereinkommens von Paris mit Eindämmung der globalen Erwärmung auf $1,5^{\circ} \mathrm{C}$ essenziell sei, um durch den Klimawandel verursachte zusätzliche Herzinfarkte zu vermeiden.

Extreme Temperaturen, insbesondere Hitze, sind aber nicht nur mit einer erhöhten Herzinfarktinzidenz verbunden, sondern auch mit einer erhöhten Rate an Todesfällen durch Herzinsuffizienz und Schlaganfälle. Dies wurde beispielsweise durch Breitner et al. für das südliche Bayern anhand einer Zeitreihenanalyse der ursachenspezifischen Mortalität zwischen 1990 und 2006 aufgezeigt (2014).

In einer Studie der Uniklinik Augsburg wurde über 10 Jahre hinweg der Zusammenhang zwischen bestimmten Wetterlagen und Schlaganfällen untersucht. Anhand von knapp 18.ooo Schlaganfall-Fällen aus der Region Augsburg - die meisten von ihnen Neuerkrankte, aber auch Patienten mit wiederholten Schlaganfällen - konnte gezeigt werden, dass sich Wetterveränderungen auf die beiden Schlaganfall-Subtypen ischämischer Schlaganfall (Hirninfarkt) und hämorrhagischer Schlaganfall (Hirnblutung) unterschiedlich auswirken (Ertl et al. 2019). So bringen trockene, warme Luftmassen ein erhöhtes Risiko für den ischämischen Hirninfarkt mit sich, der über $80 \%$ aller Schlaganfälle ausmacht. Allerdings führen sie zu einem geringeren Risiko für Hirnblutungen. Umgekehrt ist es bei trockenen, kühlen Luftmassen: Sie befördern Hirnblutungen, ziehen aber ein selteneres Auftreten von ischämischen Hirninfarkten nach sich. Auch bei feuchten Luftmassen konnte ein verringertes Auftreten von Hirninfarkten nachgewiesen werden.

Betrachtet man die Temperaturentwicklung im Zeitraum weniger Tage vor dem Schlaganfallereignis, so findet man auch hier differenzierte Einflüsse auf die Schlaganfalls- oder Blutungshäufigkeit, die pathophysiologisch allerdings noch nicht vollends geklärt sind.

\subsection{Gefährdete Bevölkerungsgruppen}

Zu den besonders gefährdeten Bevölkerungsgruppen während Hitzeperioden gehören neben denjenigen mit erhöhtem Lebensalter insbesondere diejenigen mit Komorbiditäten. Wetterbedingte Zusammenhänge mit erhöhten Notfalleinweisungen ins Krankenhaus, sowohl durch Kälte, als auch durch Hitze bedingt, sind z.B. bei Patienten mit Diabetes beschrieben, wohingegen erhöhte Notfalleinweisungen in Zusammenhang mit Bluthochdruck (in der Regel Hochdruckkrisen) dagegen eher mit Kälteeinbrüchen als mit Hitzewellen assoziiert sind (Bai et al. 2016).

Bei Diabetikern sind insbesondere Akutmanifestationen von Herz-Kreislauf-Erkrankungen ausschlaggebend. So waren in einer Studie aus Toronto Hitzeperioden bei Diabetikern mit erhöhtem Risiko einer Hospitalisierung wegen kardiovaskulärer Ursache verbunden. Die eingeschränkte thermoregulatorische Reaktionsfähigkeit bedingt durch die autonome Dysfunktion und die besondere Empfindlichkeit auf hitzeinduzierte vermehrte endotheliale Dysfunktion und Hyperkoagulabilität scheinen hier besonders mitverursachend zu sein (Lavigne et al. 2014).

Zudem sind Patienten mit vorbestehendem Herzinfarkt oder bekannter kardiovaskulärer Grunderkrankung besonders vulnerabel für schädliche Kurzzeiteffekte von Temperaturanstiegen (Rocklov et al. 2014).

Interessanterweise sind offenbar bestehende Adaptationsmechanismen an Temperatur und Temperaturschwankungen für unterschiedliche Suszeptibilitäten verantwortlich, sodass nördlichere Länder anfälliger für Hitzewellen sind als südlicher gelegene (Liu et al. 2015).

Der bisherige Fokus des Artikels lag auf den direkten temperaturbedingten Folgen auf den menschlichen Körper. Zu berücksichtigen ist, dass nicht nur der Hitzestress, sondern auch andere durch den Klimawandel bedingte Folgen wie hohe bodennahe Ozonkonzentrationen 
oder erhöhte Feinstaubkonzentrationen während der Hitzeperioden für die erhöhte herzkreislaufbedingte Morbidität und Mortalität verantwortlich sind. So begünstigt Feinstaub in erster Linie Gefäßschäden und damit Krankheiten wie Herzinfarkt, Schlaganfall, Herzrhythmusstörungen und Herzschwäche (Münzel et al. 2017). Auf die Interaktion von Temperatur und Luftschadstoffen wird in Kapitel 8 dieses Buches näher eingegangen.

\subsection{Maßnahmen zur Abmilderung der klimabedingten Auswirkungen auf die Herz-Kreislauf-Morbidität und -Mortalität}

\subsubsection{Allgemeine Maßnahmen}

Bundes-/landespolitisch: Hierzu heißt es in den 2017 vom Bundesministerium für Umwelt, Naturschutz und nukleare Sicherheit herausgegebenen Handlungsempfehlungen: „Abhilfe kann hier - neben den weltweiten Bemühungen zur Bekämpfung des Klimawandels - nur durch koordinierte Aktivitäten zur Prävention von Hitzeauswirkungen auf die Bevölkerung geschaffen werden. Hierzu eignen sich Hitzeaktionspläne auf der Grundlage der Empfehlungen der Weltgesundheitsorganisation (WHO), die den Handlungsbedarf formulieren und Vorgaben machen, auf welcher Ebene und von welcher Stelle welche Maßnahmen umgesetzt werden sollen. Konkrete Pläne sollten auf die jeweilige Region abgestimmt sein." (BMU 2017). Kritisiert wird allerdings, dass diese aber bisher auf regionaler und lokaler Ebene nicht systematisch umgesetzt wurden.

Verbandspolitisch: Beispielsweise fordert die Bundesärztekammer (BÄK) Länder und Kommunen auf, konkrete Maßnahmenpläne für Kliniken, Not- und Rettungsdienste sowie Pflegeeinrichtungen zur Vorbereitung auf Hitzeereignisse $z u$ entwickeln.

Fachkräfte im Gesundheitswesen haben eine wichtige Funktion bei der Durchsetzung rascherer
Fortschritte bei der Bekämpfung der gesundheitlichen Folgen des Klimawandels, da sie darin ausgebildet sind, Patienten über Gesundheitsgefahren aufzuklären. Sie können die Gesundheitsrisiken, die durch den Klimawandel entstehen, besser kommunizieren und dementsprechend möglicherweise auch die Politik überzeugen, dass Treibhausgasemissionen reduziert werden müssen (Watts et al. 2015).

Ein weiterer wichtiger Ansatzpunkt ist die vermehrte und strukturierte Vermittlung der Zusammenhänge zwischen Klimawandel und Gesundheit in der Ausbildung von Angehörigen der Heilberufe (Ärzte, Pflegekräfte und andere Angehörige von Gesundheitsberufen) durch Inkorporierung in die Ausbildungskurrikula/Lehrpläne und im Rahmen von Fortbildungsveranstaltungen.

\subsubsection{Konkrete präventivmedizinische Handlungsempfehlungen und Strategien}

Die Deutsche Allianz Klimawandel und Gesundheit (KLUG) hat unter dem Eindruck der aktuellen Corona-Pandemie in Zusammenarbeit mit Experten vom Helmholtz Zentrum München und dem Universitätsklinikum Heidelberg in einer ad hoc Arbeitsgruppe vier Informationsblätter herausgegeben, die praxisnahe Handlungsempfehlungen an Gesundheitsdienstleister, aber auch potenziell Betroffene erarbeiteten (abrufbar unter www.hitzezozo.de).

Generell sollten Angehörige des Gesundheitswesens auf Risiken aufmerksam machen und auch jene Maßnahmen stärken, die sowohl klimafreundlich sind als auch die Gesundheit der Risikogruppen fördern (Co-Benefits). Wichtig ist die Identifikation und Beratung nicht nur von morbiditätsbedingten Risikopatienten, sondern auch von Risikopatienten, die in ihrer Wärmeregulation und/oder Verhaltensanpassung eingeschränkt sind, z.B. mit ungünstigen Wohnverhältnissen (städtische 
II Gesundheitliche Auswirkungen des Klimawandels und Herausforderungen

für die medizinische Versorgung in Deutschland

Wärmeinsel, Dachwohnung etc.). Da Arzneimittel durch hohe Temperaturen ihre Wirksamkeit verlieren können, sind zudem auch Hinweise auf die empfohlene Lagerungstemperatur (maximal $25^{\circ} \mathrm{C}$ ) bedeutsam.

\subsection{Herausforderungen für die haus-/ fachärztliche Versorgung durch Arzneimittelwechselwirkungen}

Die Vermittlung von Wissen über gefährliche Arzneimittelnebenwirkungen ist - neben dem Aussprechen von und Sensibilisieren für Hitzeschutzmaßnahmen - eine der wichtigsten unmittelbaren Maßnahmen zur Abwendung von hitzebedingten Gesundheitsschäden. Entsprechende Maßnahmen richten sich an alle Angehörige von Gesundheitsfachberufen, insbesondere an Ärzte, aber auch an Pflegekräfte und Risikopatienten selbst (s. Kap. 4.3). Gerade Patienten mit bereits durchgemachten Ereignissen, wie z.B. Herzinfarkt, Schlaganfall, Herzschwächeepisoden oder diejenigen mit Risikofaktoren hierfür (Diabetes, Bluthochdruck, Adipositas, Hypercholesterinämie, Raucher), leiden auch oft an anderen Erkrankungen (Polymorbidität) bzw. nehmen gegen ihre Risikofaktoren/Herz-Kreislauf-Erkrankungen zahlreiche Medikamente ein.

Einige dieser Medikamente haben ein umfassendes Interaktionspotenzial, welches durch die Einwirkungen von Hitze massiv gesteigert werden kann. Deshalb ist es besonders wichtig, diese Patienten zu identifizieren und hinsichtlich ihrer Medikamenteneinnahme streng zu überwachen. Hier kommt insbesondere den Hausärzten, aber auch allen Fachärzten, die indikationsspezifische Medikamente verschreiben, hohe Verantwortung zu. Aber auch Angehörige anderer Gesundheitsberufe (z.B. in Pflegeeinrichtungen) sind aufgefordert, bei Hitzeperioden die sonst übliche „Medikamentenration“ kritisch zu hinterfragen und ggf. einen Mediziner hinzuzuziehen.
Tabelle 1 gibt für einzelne Wirkstoffklassen Beispiele für mögliche Nebenwirkungen.

Es gibt zweierlei mögliche Wechselwirkungen:

- Zum einen können Medikamente, wenn relativ überdosiert (z.B. bei vorwiegend über die Niere ausgeschiedenen Medikamenten bei exsikkosebedingter Nierenschwäche) oder durch Hitze in ihrer Wirkung verstärkt Herz-Kreislauf-Notfälle induzieren, z.B. durch das Auslösen von Hochdruckkrisen.

- Zum anderen bewirken die durch Hitzeeinwirkung reduzierten Adaptationsmechanismen des Körpers ein vermehrtes Nebenwirkungspotenzial.

Mit an vorderster Stelle sind hier alle Arzneimittel mit anticholinerger Wirkung zu nennen. Diese sind besonders gefährlich, da sie die zentrale Temperaturregulierung hemmen, was in Hitzephasen den wichtigen Ausgleichsmechanismus des Schwitzens unterbindet. Diese anticholinerge Wirkung ist in vielen Arzneimitteln enthalten, die wiederum für ein breites Indikationsspektrum eingesetzt werden, an die man a priori nicht so schnell denkt!

Herz-Kreislauf-Mittel per se bergen - in der Regel weniger aufgrund anticholinerger Eigenschaften - ein hohes Risiko einer unerwünschten Nebenwirkung, gerade bei hitzebedingten körperlichen Adaptationsmechanismen. So kann die hitzebedingte Vasodilatation den blutdrucksenkenden Effekt vieler Herz-KreislaufMittel deutlich verstärken mit der Folge von Synkopen (Bewusstseinsverlust) mit möglicherweise schwerer Verletzungsfolge oder kritischen Organischämien bis hin zum Herzinfarkt. Besonders gefährdet sind hier alle Patienten mit systolisch bedingter Herzinsuffizienz, da diese Wirkstoffe bekommen, die auch Hochdruckpatienten erhalten (Antihypertensiva). Antihypertensiva - auch als Herzinsuffizienzmedikamente eingesetzt - bergen allerdings per se schon die Gefahr einer zu starken Blutdrucksenkung. Besonders gefährlich sind in diesem $\mathrm{Zu}-$ sammenhang Diuretika. Sie werden einerseits 
4 Der Einfluss des Klimawandels auf das Auftreten von Herz-Kreislauf-Erkrankungen.

Handlungsansätze und die besondere Herausforderung durch Arzneimittelwechselwirkungen

Tab. 1 Medikamenteninteraktion und Nebenwirkungspotenzial in Hitzeperioden bei ausgewählten Wirkstoffen bzw. Indikationsklassen

\begin{tabular}{|c|c|c|}
\hline $\begin{array}{l}\text { Wirkstoff/Indikations- } \\
\text { klasse }\end{array}$ & Beispiele & $\begin{array}{l}\text { Art der durch Hitze aggravierten } \\
\text { Nebenwirkung }\end{array}$ \\
\hline $\begin{array}{l}\text { anticholinerge } \\
\text { Arzneimittel } \\
\text { (dazu zählen viele } \\
\text { Psychopharmaka, } \\
\text { Antidepressiva, aber } \\
\text { auch Bronchoinhalativa } \\
\text { u.a.) }\end{array}$ & $\begin{array}{l}\text { viele Benzodiazepine (Schlafmittel), Atosil } \\
\text { (Antipsychotikum), trizyklische Antidepressiva, } \\
\text { Ipra/Tiotropiumbromid (COPD), Trospiumchlorid, } \\
\text { (gegen Dranginkontinenz), Biperiden, } \\
\text { Oxybutinin (Antiparkinsonmittel), Butylscopala- } \\
\text { min (Magen-Darm-Krämpfe), Scopolamin (gegen } \\
\text { Übelkeit z.B. bei Reisekrankheit), Dimenhydrinat } \\
\text { (gegen Übelkeit, zur Beruhigung), Antihistamini- } \\
\text { ka (gegen Allergien) }\end{array}$ & $\begin{array}{l}\text { Hemmung der zentralen Temperatur- } \\
\text { regulierung } \rightarrow \text { Schwitzen verringert, } \\
\text { Kognitionseinschränkung; Blutdruck- } \\
\text { abfälle und andererseits Blutdruckkrisen }\end{array}$ \\
\hline \multirow[t]{2}{*}{$\begin{array}{l}\text { Antihypertensiva (AHT) } \\
\text { (Bluthochdruckmittel) }\end{array}$} & $\begin{array}{l}\text { Betablocker, ACE-Hemmer, Sartane, Kalzium- } \\
\text { antagonisten, Clonidin, Monoxidin (letztere } \\
\text { zentral wirksam) }\end{array}$ & $\begin{array}{l}\text { einerseits Verhinderung der Erweiterung } \\
\text { der Blutgefäße (Betablocker) in der Haut } \\
\rightarrow \text { Hitzeableitung durch Konvektion } \\
\text { verringert, Erhöhung der Schweiß- } \\
\text { sekretionsschwelle (zentrale AHT) }\end{array}$ \\
\hline & & $\begin{array}{l}\text { andererseits Verstärkung der blutdruck- } \\
\text { senkenden Wirkung durch Vasodilatation } \\
\rightarrow \text { Blutdruckabfälle }\end{array}$ \\
\hline $\begin{array}{l}\text { Diuretika (als Hoch- } \\
\text { druckmittel oder bei } \\
\text { Herzinsuffizienz) }\end{array}$ & $\begin{array}{l}\text { Hydrochlorothiazid (HCT), in vielen Kombina- } \\
\text { tionspräparaten enthalten!, Chlortalidon, } \\
\text { Indipamid, Torasemid, Furosemid, Spironolacton }\end{array}$ & $\begin{array}{l}\text { Verstärkung der Blutdrucksenkung, } \\
\text { Austrocknung, Nierenversagen, } \\
\text { gefährliche Elektrolytentgleisung }\end{array}$ \\
\hline $\begin{array}{l}\text { Antianginosa } \\
\text { (bei koronarer } \\
\text { Herzerkrankung) }\end{array}$ & Nitrate wie ISMN, ISDN, Molsidomin & $\begin{array}{l}\text { besondere gefäßerweiternde Wirkung } \rightarrow \\
\text { gefährliche Blutdruckabfälle }\end{array}$ \\
\hline Antiepileptika & $\begin{array}{l}\text { Carbamazepin, Valproinsäure, Lamotrigin, } \\
\text { Benzodiazepine, Barbiturate }\end{array}$ & $\begin{array}{l}\text { Beeinträchtigung der kognitiven } \\
\text { Wachsamkeit, Verstärkung von Elektrolyt- } \\
\text { entgleisungen (Hyponatriämie) }\end{array}$ \\
\hline Schmerzmittel & $\begin{array}{l}\text { NSAR (Ibuprofen, Diclofenac, Coxibe), Opiate } \\
\text { (Fentanylpflaster), orale Medikamente wie } \\
\text { Morphin, Hydromorphon, Codein, Dihydroco- } \\
\text { dein, Pethidin, Tilidin, Tramadol }\end{array}$ & $\begin{array}{l}\text { Gefahr des Nierenversagens, Auslösung } \\
\text { von Hochdruckentgleisungen, Verschlech- } \\
\text { terung von Herzinsuffizienz bei Exsiccose, } \\
\text { bei Opiaten Gefahr der Akkumulation bzw. } \\
\text { verstärkten Wirkstofffreisetzung bei } \\
\text { transdermalen Systemen } \rightarrow \text { kognitive } \\
\text { Beeinträchtigung, Atemdepression, } \\
\text { anticholinerge Nebenwirkungen }\end{array}$ \\
\hline Insuline & Basalinsuline, schnell wirksame Insuline etc. & $\begin{array}{l}\text { unter Hitzebedingungen ggf. rascheres } \\
\text { Anfluten mit Hypoglykämiegefahr }\end{array}$ \\
\hline
\end{tabular}

zur Blutdrucksenkung, gern auch in Kombination mit andern Antihypertensiva eingesetzt, insbesondere aber zur Entwässerung bei Herzinsuffizienten. Deshalb sind sie in Tabelle 1 se- parat aufgerührt. Denn neben einer überschießenden Blutdrucksenkung kann es bei Hitze durch vermehrt auftretende Exsikkose (Austrocknung) zu Nierenversagen mit möglicher 
II Gesundheitliche Auswirkungen des Klimawandels und Herausforderungen

für die medizinische Versorgung in Deutschland

Wirkstoffakkumulation bei polypharmazeutisch behandelten Patienten kommen. Darüber hinaus besteht hier die Gefahr sogenannter Elektrolytentgleisung (Hyper- wie Hypokaliämie, Hypo-, Hypernatriämie) mit sehr schwerwiegenden Folgen (Induktion bösartiger Herzrhythmusstörungen, dadurch ausgelöster plötzlicher Herztod, komatöse Zustände). Gerade Patienten mit Herzinsuffizienz sind wegen ihrer Grunderkrankung und der bestehenden Polypharmazie bei vermehrter Hitzeexposition und den noch hinzukommenden toxischen Effekten von Feinstaub und Ozon besonders gefährdet. So kann es z.B. auch zu einer unerwünschten Wirkung von Antiarrhythmika kommen, die diese Patienten oft einnehmen, sodass sie einer besonderen Überwachung bedürfen (vermehrte EKG-Kontrollen zur Bestimmung der sog. QT-Zeit, Blutabnahmen zur Beurteilung der Nierenfunktion und des Elektrolythaushaltes) (s. Fallbeispiel). Antianginosa (z.B. Nitrate, Molsidomin, Ranolazin) erhalten viele Patienten mit Angina pectoris (Brustenge) bei koronarer Herzkrankheit. Diese können durch ihre vasodilatierenden Eigenschaften negative Folgen entwickeln. Diese Medikamente sollten bei gefährdeten Patienten in einer Hitzewelle vorrangig abgesetzt werden, da diese Wirkstoffe auch keinerlei prognostische Wirkung hinsichtlich harter Endpunkte haben.

Eine zunehmende Anzahl von Patienten nimmt zeitlich beschränkt oder chronisch Schmerzmittel ein. Auch diese bergen zum Teil erhebliche Gefährdungen unter entsprechenden Temperaturbedingungen, insbesondere ist hier von transdermal verabreichten Opiaten zu warnen.

Diabetiker gehören per se zur Risikogruppe. Besondere Vorsicht ist geboten bei insulinabhängigen Patienten, da sich gerade bei starkem Schwitzen und Änderungen des Hautturgors die Resorption des Insulins stark ändern kann.

In Tabelle 1 nicht separat aufgeführt, da auch nicht einheitlich abzubilden, sind pflanzliche Wirkstoffe/naturheilkundliche Präpa- rate. Diese werden häufig ,vergessen“ und vom Patienten nicht an den Arzt berichtet. Auch wenn über diesbezügliche Wechselwirkungen wenig bekannt ist, sollten auch diese Präparate immer mitbedacht werden. Gerade alkaloidhaltige pflanzliche Substanzen oder Johanniskraut haben bekanntlich medikamentöses Interaktionspotenzial.

\subsection{Mögliche Strategien und Handlungs- empfehlungen über die Umsetzung von Hitzeaktionsplänen hinaus}

Welche weiteren Strategien neben den bereits oben skizzierten Maßnahmen wie allgemeine Handlungsempfehlungen zur Umsetzung von Hitzeaktionsplänen und deren Anwendung sind denkbar? So wäre die Entwicklung und Implementierung eines Smartphone-basierten Ampelsystems in Form einer App empfehlenswert. Vorstellbar wäre, dass sich möglichst viele der Risikopatienten bzw. deren Angehörige sowie die behandelnden Ärzte (und Pflegeeinrichtungen) registrieren bzw. eine zu programmierende App (analog der derzeit entwickelten Corona-Warn-App) nutzen. Ähnlich wie die Warnungen des Deutschen Wetterdienstes können in der App Vorhersagen zu gesundheitsgefährdenden Witterungsbedingungen, wie z.B. hohe Temperaturen, Luftfeuchtigkeit oder fehlende Nachtabkühlung, angezeigt werden. Darüber hinaus sollte die App den Anwender über zu treffende Hitzeschutzmaßnahmen informieren. Mittels der vorgeschlagenen App können zudem auch Checklisten bereitgestellt werden, die die Behandlung mit bzw. Einnahme von kritischen Medikamenten abfragen. Dabei sollte auch eine direkte Eingabe der eingenommenen Medikamente/Wirkstoffe möglich sein. Die Anwendung soll anzeigen, ob eine kritische Wirkstoffgruppe darunter ist, mit dem Hinweis, unbedingt den behandelnden Arzt zu konsultieren, ob und in welcher Dosierung die Medikamente weiter eingenommen werden sollen. 


\subsection{Fazit}

Der Zusammenhang zwischen dem Auftreten von Hitzewellen und der Häufigkeit von Herzinfarkten oder anderer Kreislauferkrankungen wie Schlaganfall oder vermehrter Einweisung wegen Herzinsuffizienz oder Hochdruckentgleisungen ist hinreichend belegt. Präventive Maßnahmen zum Schutz der Bevölkerung vor hitzebedingten Gesundheitsschäden können durch koordinierte Aktivitäten, wie z.B. verbesserte Edukation über die Auswirkungen von Hitzeperioden auf die Gesundheit in der Ausbildung und Vermittlung in Gesundheitsfachberufen, getroffen werden. Besonders gefährdet sind Risikogruppen, einerseits aufgrund ihrer Grunderkrankung und andererseits insbesondere durch während Hitzeperioden auftretender verstärkter Arzneimittelnebenwirkungen, da gerade diese Patienten häufig mit mehreren Medikamenten behandelt werden. Hierbei könnten gezielte Maßnahmen greifen: Die Implementierung eines Smartphone-basierten Ampelsystems mit rechtzeitiger Warnung aller Risikopatienten bzw. deren Angehöriger bzw. Ärzte und Übermittlung allgemein zu treffender Hitzeschutzmaßnahmen sowie Abfrage kritischer Medikamente.

\section{Literatur}

Bai L, Li Q, Wang I, Lavigne E, Gasparrini A, Copes R (2016) Hospitalizations from Hypertensive Diseases, Diabetes, and Arrhythmia in Relation to Low and High Temperatures: Population-Based Study Sci Rep 6:30283

Breitner S, Wolf K, Peters A, Schneider A (2014) Short-term effects of air temperature on cause-specific cardiovascular mortality in Bavaria, Germany Heart 100(16):1272-80
Bundesministerium für Umwelt, Naturschutz und nukleare Sicherheit (BMU) (2017) Handlungsempfehlungen für die Erstellung von Hitzeaktionsplänen zum Schutz der menschlichen Gesundheit. Bonn. URL: https://www.bmu.de/fileadmin/ Daten_BMU/Download_PDF/Klimaschutz/hap_handlungsempfehlungen_bf.pdf (abgerufen am 09.02.2021)

Chen K, Breitner S, Wolf K, Hampel R, Meisinger C, Heier M, von Scheidt W, Kuch B, Peters A, Schneider A; KORA Study Group (2019a) Temporal variations in the triggering of myocardial infarction by air temperature in Augsburg, Germany, 19872014. Eur Heart | 40:1600-8

Chen K, Breitner S, Wolf K, Rai M, Meisinger C, Heier M, Kuch B, Peters A, Schneider A, on behalf of the KORA Study Group (2019b) Projection of temperature-related myocardial infarction in Augsburg, Germany: moving on from the Paris Agreement on Climate Change. Dtsch Arztebl Int 116:521-7

Deutscher Wetterdienst (2020) Nationaler Klimareport: Klima Gestern, heute und in der Zukunft. URL: https://www.dwd. de/DE/leistungen/nationalerklimareport/report.html (abgerufen am 09.02.2021)

Ertl M, Beck C, Kühlbach B, Hartmann I, Hammel G, Straub A, Giemsa E, Seubert S, Philipp A, Traidl-Hoffmann C, Soentgen I, Jacobeit J, Naumann M (2019) New Insights into Weather and Stroke: Influences of Specific Air Masses and Temperature Changes on Stroke Incidence. Cerebrovasc Dis 47(5-6):275284. doi: 10.1159/000501843

Jacob D, Petersen |, Eggert B et al. (2014) EURO-CORDEX: new highresolution climate change projections for European impact research. Reg Env Chang 14:563-78

Lavigne E, Gasparrini A, Wang X, Chen H, Yagouti A, Fleury MD, Cakmak S (2014) Extreme ambient temperatures and cardiorespiratory emergency room visits: assessing risk by comorbid health conditions in a time series study. Environ Health 13(1):E48-58

Liu C, Yavar Z, Sun Q (2015) Cardiovascular response to thermoregulatory challenge. Am I Physiol Heart Circ Physiol 309(11): H1793-812

Münzel T, Sørensen M, Gori T, Schmidt FP, Rao X, Brook FR, Chen LC, Brook RD, Rajagopalan S (2017) Environmental stressors and cardio-metabolic disease: part II - mechanistic insights. Eur Heart I 38:557-564

Rocklov J, Forsberg B, Ebi K, Bellander T (2014) Susceptibility to mortality related to temperature and heat and cold wave duration in the population of Stockholm County, Sweden. Glob Health Action 7:22737

Watts N, Adger WN, Agnolucci P et al. (2015) Health and climate change: policy responses to protect public health. Lancet 386:1861-914 
II Gesundheitliche Auswirkungen des Klimawandels und Herausforderungen für die medizinische Versorgung in Deutschland

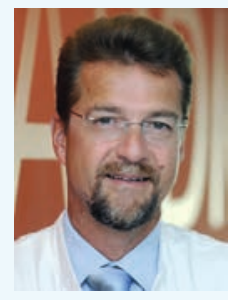

\section{Prof. Dr. med. Bernhard Kuch}

Medizinstudium in Regensburg und München, Ausbildung zum Internisten, Intensivmediziner und Kardiologen am Uniklinikum Augsburg. Wissenschaftliche Ausbildung in Münster/Westfalen am Institut für Epidemiologie und Sozialmedizin, Habilitation und Professur an der LMU München, Oberarzt und Leiter der Katheterlabore am Klinikum Augsburg bis 2011. Seit 2011 Aufbau einer kardiologischen Klinik mit Intensivstation am Stiftungskrankenhaus Nördlingen, Donau-Ries-Kliniken, seit 2016 Chefarzt und Direktor der vereinten kardiologischen und Internistischen Klinik und seit 2020 ärztlicher Direktor. Mitglied im wissenschaftlichen Beirat der Deutschen Herzstiftung, Mitglied Expertengruppe Herzkatheter/PCI-Qualitätssicherung am IQTIG. 